\title{
In praise of bats
}

\author{
Biologists have long been captivated by bats, whose unique adaptations are wonders of evolution. We \\ examine some of the many reasons why they are so important to ecologists and evolutionary biologists.
}

This issue of Nature Ecology \& Evolution features two Articles that focus on bats, so it seems an appropriate point to celebrate the importance of Chiroptera, the second largest mammalian order. With over 1,300 species, bats illustrate many key issues in ecology and evolution, from the beauty of their unique biology to their importance for ecosystem services, disease and conservation.

Let's start near the beginning. Bats are best known for being the only mammals that can truly fly; a dramatic example of evolutionary convergence with birds and pterosaurs. They are also known for their linked ability to echolocate; something that most bat species do using signals produced in their larynges. However, the pteropid bats lack this ability, instead using simpler tongue clicks in a similar way to that used by the few species of birds and terrestrial mammals that can echolocate and also by some blind humans. Because laryngeal echolocation appears not to be monophyletic, it has been unclear whether it arose more than once, or arose once and was then lost in pteropids. In this issue, Zhang and colleagues (article no. 0021 ) offer a resolution to this debate on early bat evolution by focusing on developmental biology in a phylogenetic context. They show that the cochleae of all bats develop in early fetal stages in a rapid way that is consistent with echolocation, which is in contrast to the cochlear development of other, non-echolocating, mammals. However, after this initial burst, development then slows dramatically in pteropids, suggesting they have lost the ability to echolocate.

Also in this issue, Dávalos and colleagues (article no. 0026) use bats to illustrate an important principle in island biogeography and conservation. Using one of the best fossil records there is for bats, the authors examine colonization-speciation-extinction dynamics on the Greater Antilles to work out whether the species assemblage is governed by equilibrium dynamics. It is only by incorporating data from both fossil and extant species that they are able to demonstrate equilibrium, and highlight the fact that recent anthropogenic extinctions have disturbed that equilibrium. Further modelling work predicts that at least eight million years would be needed to return to

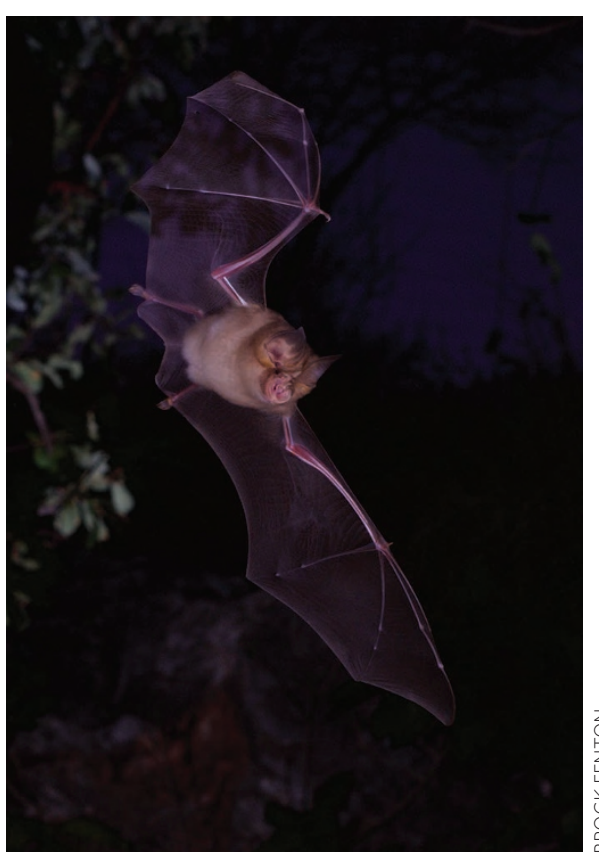

sensitivity to mortality caused by wind turbines, either due to the damaging effects of pressure change or actual direct impact. It is probably fair to say that more data are needed on this issue, especially if it is to be meaningfully incorporated as one of the many factors that need to be considered in planning energy strategy. The other concern is of more undeniable severity: the fungal disease white-nose syndrome is currently the cause of major declines in North American bat species. Probably introduced from Europe or Asia, the disease causes about $80 \%$ mortality and could lead to species extinctions. Given the ecosystem services provided by bats, it is also likely to have a strongly detrimental effect on North American agriculture.

However, diseases affecting Chiroptera are not the only reason this order is well known to epidemiologists. Bats are notorious reservoirs for zoonotic pathogens, including rabies and SARS. This is probably to do with several attributes of their life histories and their interactions with humans. Bats are long-lived and can be highly social, and they can thrive in a wide range of habitats and interact often with livestock. A greater understanding of bat biology can only help in preventing the spread of zoonotic pathogens in a world where human and wild environments are increasingly closely connected. The link between bats and human disease should certainly not be used as an excuse for culls that are not supported by scientific evidence.

The propensity of bats to live in humandominated environments and their tendency towards social living are perhaps best illustrated by the largest urban bat colony in the world. The Ann W. Richards Congress Avenue Bridge in Austin, Texas is home to about a million Mexican freetailed bats (Tadarida brasiliensis). Readers who attended either the Ecological Society of America conference in 2011 or the Evolution 2016 conference in Austin may have been lucky enough to witness their dramatic dusk-time feeding flight from the bridge. We suggest that readers who are heading to the Society for Molecular Biology \& Evolution meeting (http://www. smbe2017.org/) in Austin this July also embrace the beauty of bats. 